\title{
Feasibility of a novel non-invasive swab technique for serial whole-exome sequencing of cervical tumors during chemoradiation therapy
}

Julianna K. Bronk ${ }^{1}$, Chiraag Kapadia ${ }^{1}$, Xiaogang Wu², Bhavana V. Chapman ${ }^{1}$, Rui Wang $^{1}$, Tatiana V. Karpinets ${ }^{2}$, Jianhua Zhang ${ }^{2}$, Ann H. Klopp ${ }^{1 *}$, Lauren E. Colbert ${ }^{1 *}$

${ }^{1}$ Department of Radiation Oncology, The University of Texas MD Anderson Cancer Center, Houston, Texas; ${ }^{2}$ Department of Genomic Medicine, The University of Texas MD Anderson Cancer Center, Houston, Texas

*Shared Corresponding Authorship

Corresponding Authors: Lauren E. Colbert, MD MSCR, Department of Radiation Oncology, Unit 1052, The University of Texas MD Anderson Cancer Center, 6565 MD Anderson Blvd, Houston, TX 77030-4009; Icolbert@mdanderson.org; and Ann H. Klopp, MD PhD, Department of Radiation Oncology, Unit 1422, The University of Texas MD Anderson Cancer Center, 1400 Pressler St., Houston, TX 77030-4008; aklopp@mdanderson.org.

Running Title: Feasibility of Mutation profiling during cervical cancer CRT

Keywords: cervical cancer, chemoradiation, whole exome sequencing, noninvasive biopsy 
medRxiv preprint doi: https://doi.org/10.1101/2022.02.23.22271256; this version posted February 24, 2022. The copyright holder for this preprint

(which was not certified by peer review) is the author/funder, who has granted medRxiv a license to display the preprint in perpetuity.

All rights reserved. No reuse allowed without permission.

Funding: This study was funded in part by the Radiological Society of North America

(RSNA) Resident/Fellow Award (LEC), National Institutes of Health (NIH) through MD

Anderson's Cancer Center Support Grant P30CA016672 (LEC, AHK), and The

University of Texas MD Anderson Cancer Center HPV-related Cancers Moonshot (LEC, AHK).

Conflicts of interest: The authors declare no potential conflicts of interest. 


\section{Abstract}

Background: Clinically relevant genetic predictors of radiation response for cervical cancer are understudied due to the morbidity of repeat invasive biopsies required to obtain genetic material. Thus, we aimed to develop a novel noninvasive cervical swab technique to collect tumor DNA with adequate throughput to perform whole-exome sequencing (WES) at serial time points over the course of chemoradiation therapy (CRT).

Methods: Cervical cancer tumor samples from patients undergoing chemoradiation were collected at baseline, at week 1, week three, and at the completion of CRT (week 5) using a noninvasive swab-based biopsy technique. Swab samples were analyzed with whole-exome sequencing (WES) with mutation calling using a custom pipeline optimized for shallow whole-exome sequencing with low tumor purity. Tumor mutation changes over the course of treatment were profiled.

Results: 217 samples were collected and successfully sequenced for 70 patients. A total of 33 patients had a complete set of samples at all four time points. The mean mapping rate was $98 \%$ for all samples, and the mean target coverage was 180 . Overall mutation frequency decreased during CRT with disease response but mapping rate and mean target coverage remained at $>98 \%$ and $>180$ reads at week 5 .

Conclusion: This study demonstrates the feasibility and application of a noninvasive swab-based technique for WES analysis to investigate dynamic tumor mutational changes during treatment and may be a valuable approach to identify novel genes which confer radiation resistance. 


\section{Introduction}

The global burden of cervical cancer is growing despite the development of HPV vaccines aimed at disease prevention ${ }^{1}$. In 2018, approximately 570,000 new cases of cervical cancer were diagnosed worldwide, resulting in more than 311,000 deaths $^{2}$. High-risk human papillomaviruses (HPVs) are essential in cervical dysplasia and carcinogenesis and cause most cervical cancers ${ }^{3}$. Multimodality therapy is the standard of care for treating locally advanced disease and involves daily external beam radiation treatment, brachytherapy, and weekly chemotherapy ${ }^{4}$. The rate of tumor regression during chemoradiotherapy (CRT) is variable and is strongly associated with survival ${ }^{5,6}$; however, predictive markers of radiation treatment sensitivity and resistance are currently unknown.

Whole-exome sequencing (WES) technology is a powerful tool that allows for comprehensive analysis of the frequency of somatic mutations, overall tumor mutational burden, and single nucleotide human exome variants (SNVs), which can lead to the identification of pathways that may be functionally significant in cancer outcomes ${ }^{7}$.

Previous comprehensive studies of cervical cancer genomics have relied on the analysis of untreated tumors, and none have identified predictors of radiation response $e^{8,9}$. This is mainly due to the challenges of repeated biopsy sampling over the course of the therapy, which are logistically challenging to acquire and entail significant patient morbidity. Nevertheless, cervical tumors are an ideal setting for the serial study of treatment response because tumors can be readily monitored by physical exam and are accessible for sampling through the course of chemoradiotherapy. Encouragingly, successful non-invasive swab-based sample acquisition of DNA has been 
demonstrated in the screening and diagnosis of precancerous and cancerous lesions in the oral cavity and detection of HPV-associated cutaneous lesions by PCR ${ }^{10-13}$.

This study hypothesizes that non-invasive swab-based sampling of cervical tumors can serve as a robust noninvasive method to acquire tumor specimens for WES. Using swab-based biopsies collected prospectively in 70 patients undergoing CRT for newly diagnosed cervical cancer, we: (1) acquire tumor DNA samples adequate for WES in the majority of patients and (2) develop a custom pipeline optimized for shallow WES with low tumor purity to facilitate mutation calling. 


\section{Materials and Methods}

\section{Patient population and treatment characteristics}

Patients were enrolled in an IRB-approved (2014-0543) multi-institutional prospective clinical trial at the The University of Texas MD Anderson Cancer Center and the Harris Health System, Lyndon B. Johnson General Hospital Oncology Clinic (Figure 1A, Table 1). Inclusion criteria were newly diagnosed cervical cancer per the Federation of Gynecology and Obstetrics (FIGO) 2009 staging system, clinical stage IB1-IVA cancers, and visible, an exophytic tumor on speculum examination with planned definitive treatment of intact cervical cancer with external beam radiation therapy, cisplatin, and brachytherapy. Patients with any previous pelvic radiation therapy were excluded.

Patients underwent standard-of-care pretreatment evaluation for disease staging, including tumor biopsy to confirm the diagnosis; pelvic magnetic resonance imaging (MRI) and positron emission tomography/computed tomography (PET/CT); and standard laboratory evaluations, including a complete blood cell count, measurement of electrolytes, and evaluation of renal and liver function. Patients received pelvic radiation therapy to a total dose of $40-45$ Gy delivered in daily fractions of 1.8 to 2 Gy over 4 to 5 weeks. Thereafter, patients received intracavitary brachytherapy with pulsed-dose-rate or high-dose-rate treatments. According to standard institutional protocol, patients received cisplatin ( $40 \mathrm{mg} / \mathrm{m} 2$ weekly) during external beam radiation therapy. Patients underwent repeat MRI at the completion of external beam radiation therapy or at the time of brachytherapy, as indicated by the extent of disease. Patients with no residual tumor on repeat MRI were considered to be exceptional responders, while those with 
residual MRI tumor volumes $\leq 20 \%$ and $>20 \%$ of initial volumes after 4 to 5 weeks after initiation of RT were considered to be standard and poor responders, respectively.

\section{Sample collection and DNA extraction}

Isohelix swabs (product \# DSK-50 and XME-50, www.isohelix.com, UKSamples) were brushed against the viable cervical tumor several times by a clinician from the department of radiation oncology or gynecologic oncology at either The University of Texas MD Anderson Cancer Center or Lyndon B. Johnson General Hospital Oncology Clinic. The isohelix swab has a unique matrix design that yields one to five micrograms of high-quality DNA sufficient for sequencing applications from a single swab of the tumor surface ${ }^{14}$. Patients underwent swabbing at baseline, the end of week 1 (after five fractions), at the end of week 3 (after 10-15 fractions), and within a week before the first brachytherapy treatment or at the time of brachytherapy (week 5), for a total of four swabs during radiation therapy (Figure 1A). In each swabbing session, attention was taken to obtain samples from the same general tumor region. Normal buccal samples were collected once at baseline to identify germline mutations present in individual patients. DNA was extracted from normal buccal and cervical cancer samples per Isohelix \# DSK-50 manufacturer's instructions.

\section{Whole-exome sequencing and mutational analysis}

Illumina WES sequencing was performed on normal buccal control and cervical tumor DNA swab samples. Captured libraries were sequenced on a HiSeq 4000 series (Illumina Inc., San Diego, CA, USA) on a TruSeq version 3 Paired-end Flowcell 
according to manufacturer's instructions at a cluster density between 700-1000K clusters $/ \mathrm{mm}^{2}$. Sequencing was performed for $2 \times 100$ paired-end reads with a 7nucleotide read for indexes using Cycle Sequencing version 3 reagents (Illumina). The average coverage achieved with the Roche Nimblegen probes was 180 reads (range 50-359) for cervical tumor samples.

WES exome sequencing data were aligned to the human reference genome by using the Burrows-Wheeler Aligner (BWA $)^{15}$. Duplicate reads were removed by using Picard and then realigned and recalibrated. Paired-end raw sequence reads in fastq format were aligned to the reference genome (human Hg19) using Burrows-Wheeler Aligner (BWA $)^{15}$ with three mismatches with 2 in the first 40 seed regions for sequences less than 100 bp or using BWA-MEM with 31 bp seed length for sequences over 100 bp. The aligned BAM files were subjected to mark duplication, re-alignment, and recalibration using Picard and the Genome Analysis Toolkit ${ }^{16}$ before any downstream analyses.

A custom computational pipeline was optimized for mutation calling (Figure 2). Based on the alignment results (BAM files) processed above, somatic mutations, including single-nucleotide variants (SNVs) and small insertions and deletions (INDELs), were obtained through merging variants from multiple somatic variant callers - MuTect, Pindel, VarScan2, LoFreq, GATK4, Mutect2, and Strelka2 ${ }^{17,18}$. Mutations previously reported in the public database (dbSNP138, 1000Genomes, ESP6500, and EXAC) with $>1 \%$ allele frequency were removed. Next, the following mutation-filtering criteria were applied: (i) sequencing depth $\geq 20$ for tumor and $\geq 10$ for normal, (ii) tumor variant allele frequency $(\mathrm{VAF}) \geq 2 \%$, and normal VAF $<2 \%$, (iii) Evidence (number of 
medRxiv preprint doi: https://doi.org/10.1101/2022.02.23.22271256; this version posted February 24, 2022. The copyright holder for this preprint (which was not certified by peer review) is the author/funder, who has granted medRxiv a license to display the preprint in perpetuity. All rights reserved. No reuse allowed without permission.

somatic variant callers supported) $\geq 2$. Associations between somatic mutations and disease progression/treatment response were analyzed and visualized using Maftools ${ }^{19}$. Tumor purity (that is, the proportion of cancer cells in a tumor sample) was calculated from SNVs by the Tumor Purity Estimation (TPES) as well as from copy number profiles using the Sequenza method ${ }^{20}$ (Table S1). 


\section{Results}

\section{Patient and tumor characteristics}

Clinicopathologic data are summarized in Table 1. Overall, 50 patients $(71.4 \%)$ had advanced disease (stage IIB or greater), and most had squamous cell carcinoma with moderate or poor differentiation. The median tumor size (based on the short axis diameter on pretreatment MRI) was $5 \mathrm{~cm}$ (range $1.2-11.5 \mathrm{~cm}$ ). Forty-five patients had positive pelvic or para-aortic lymph nodes on PET or CT.

\section{Quality Characteristics for All Samples}

Two hundred thirty total tumor samples were collected (Figure 1). For three patients, matching normal (buccal) samples failed either DNA quality or quantity quality check (QC), and thus 12 paired tumor samples were excluded. Two hundred eighteen total tumor samples and 70 normal germline samples were sequenced. Of these, 161 (74\%) had both optimal DNA quality and quantity QC, 54 (25\%) had suboptimal quantity, but adequate quality and $2(1 \%)$ had optimal quantity but low-quality DNA. 1 sample failed sequencing due to both suboptimal DNA quality and quantity; thus, 217 total tumor samples from 70 patients were included in the analysis (Figure 1B). Median DNA concentration per sample was $12 \mathrm{ng} / \mathrm{uL}$ (range 1.5-167 $\mathrm{ng} / \mathrm{uL}$ ). The mean total reads per sample was 216 million (+/-50), and mean mapping rate was $98.15 \%(+/-1.84)$ (Table 2). Total DNA concentration by timepoint was not different by timepoint $(p=0.62)$, nor were total reads $(p=0.65)$, mapping rate $(p=0.37)$, or mean target coverage $(p=0.54)($ Figure 1C-F). No significant differences were detected by sequencing batch. 


\section{Mutation Characteristics for all Samples}

All patients had samples analyzed at baseline $(\mathrm{N}=70)$, while only 49 had samples at Week 1, 42 at Week 3, and 61 at Week 5. 33 patients had samples analyzed at all four time points. Mean number of substitutions, insertions and deletions over time was 337 (range 50-2,857) at baseline, 394 (range 48-2,281) at Week 1, 321 (range 57-2,223) at Week 3 and 160 (range 38-1,843) at Week 5 (Table 2, Table S1).

Median tumor purity of all samples as calculated by the Sequenza algorithm decreased from baseline (median 0.195 [range 0.10-0.99]) to week 5 (median 0.185 [range 0.10-0.98]; $p=0.046$; Table 2, Table S1). Median tumor purity of all samples as calculated by the TPES algorithm at baseline was 0.61 (range 0.17-0.99) and 0.55 (range 0.12-0.97) at week $5(p=0.097 ;$ Table 2, Table S1).

In all analyzed samples $(n=217)$, the top 5 most common alterations were in PIK3CA, FBXW7, FNDX1, KMT2D, and CSMD3 (Figure 3). For all 33 patients with samples available at all 4 time points ( $n=132$ samples), the top 10 most common alterations were in PIK3CA (17\%), FBXW7 (13\%), KMT2D (11\%), LRP1B (11\%), RYR2 (11\%), MUC16 (10\%), CSMD3 (8\%), EP300 (8\%), PCLO (8\%), and KMT2C (8\%)

(Figure 4). Of these, the remaining detectable alterations at week 5 were in FBXW7, LRP1B, and RYR2. From the top 50 alterations, other genes with alterations remaining at week 5 included CGREF1, CAMSAP1, DOCK11, LRP2, CRTAC1, KIF2, LTPB1, STK11, SUFU, GPR98, AGGF1, AMER1, CLTCL1, and ENC1. 


\section{Discussion}

To our knowledge, this is the first study to describe the use of non-invasive swab-based cervical tumor sample collection and performance of serial WES over the course of CRT. Tumor purity analysis confirmed the presence of tumor cells captured and sequenced at timepoints throughout the course of treatment, and gene alterations present at baseline and persistent through the course of treatment were identified. We anticipate that this methodology will allow for comprehensive characterization of changes in the mutational landscape of cervical cancers in patients undergoing treatment.

Past studies that relied on serial biopsies have been limited by logistical challenges, concerns about patient discomfort, and complications from traditional biopsies, including bleeding and infection. In a report by Weidhaas et al., gene expression profiling was performed on tissue biopsies collected from 13 patients participating in Radiation Therapy Oncology Group (RTOG) 0128 pre- and midtreatment and investigators identified a seven gene signature that predicted improved local control ${ }^{21}$. In a similar study of patients with locally advanced cervical cancer undergoing CRT, investigators acquired biopsies pretreatment and at week 3 of CRT and performed RNA-sequencing on 20 matched pairs. They found that patients who succumbed to disease at the time of their report had enrichment of gene expression from mitotic pathways and increased retention of HPV E6/E7 gene expression at week 3 of CRT, which may promote treatment resistance ${ }^{22}$. No analysis of DNA variant discovery was performed in either of these studies. Our novel, non-invasive technique allows DNA to be collected from cervical swabs, reducing obstacles to serial biopsy collections. Furthermore, while previous studies have recognized gene expression 
signatures associated with long-term patient outcomes, this is the first study to report findings from WES of cervical tumor samples collected longitudinally over the course of CRT.

As expected, tumor purity estimates for swab acquired samples were lower than previously reported estimates from the Cancer Genome Atlas (TCGA) analysis in biopsy samples $^{23}$ and decreased with disease response. While several computational methods exist to infer TP, these methods differ in the types of genomic information used, such as gene expression, SCNA, and somatic mutations ${ }^{24,25}$. To reduce the systematic bias of genomic investigation of samples containing both tumor and non-neoplastic tissue, TP levels are often considered during analysis to deconvolute contaminant contributions from the tumor microenvironment field ${ }^{26-28}$. Thus, we evaluated two purity estimation tools with differing prediction mechanisms to better understand the cellular heterogeneity within our swab acquired samples. The Sequenza tool was developed for both exome and whole-genome deep sequencing of tumor DNA and is based on a probabilistic model applied to segmented data where average depth ratio in tumor versus normal samples and allele frequency for each segment is used to estimate for cellularity and ploidy ${ }^{29}$. Investigators found that Sequenza correctly detected ploidy in samples with as low as $30 \%$ tumor content. TPES is a computational method that predicts TP from variant allelic fraction distribution of SNVs to more accurately predict TP when tumor genomes are copy-number neutral or euploid ${ }^{20}$. This tool was validated on WES data from TCGA tumor samples and enabled TP estimation in over 1,000 samples that failed TP prediction algorithms dependent on somatic copy-number alterations. TPES estimates were enriched in samples with low genomic burden, while 
SCNA-based tools similar to Sequenza were more proficient with high genomic burden cases suggesting a complementary role for these two tools in the analysis of tumor WES data.

While the clinical significance of individual mutations was not the focus of the present study, studies of a larger cohort are underway to identify signatures and mutations associated with radiation sensitivity and resistance among women with differential responses to CRT for cervical cancer. For this future work, we hypothesize that mutations that survive the initial weeks of radiation treatment may be clinically relevant drivers of radiation resistance, and we plan to focus on characterizing the clonal architecture of residual tumors to identify more granular molecular signatures predictive of treatment response. This will permit future investigations of treatment escalation or de-escalation in appropriate populations.

There are several limitations to this study that must be addressed. First, tumor samples were not available at all four time points for 37 patients due to the inability to procure samples. Second, long-term follow-up data is not available for this cohort at this time. An additional limitation of the current study is the absence of tumor biopsy samples with which to compare sequencing output to swab-acquired DNA. A previous study examining epigenome-wide DNA methylation compared sample acquisition by biopsy and isohelix brush swab for cancers in the oral cavity found no significant difference in DNA yield between tissue and brush samples and matched tissue. Isohelix brush swabs had an excellent correlation in the oral cavity ${ }^{10}$. In this prior study, mapping efficacy was over $90 \%$ for swab-acquired DNA. Investigators successfully identified potential prognostic and predictive biomarkers for malignant lesions in the oral cavity 
with high sensitivity and specificity using a comparable isohelix swab design as was used here ${ }^{10}$. While isohelix brushes have been designed and marketed for DNA acquisition of the buccal mucosa, we were able to acquire sufficient quality and quantity DNA for WES in all but one tumor sample with collected samples by isohelix brushings from cervical mucosa. This method could rapidly be applied to the study of other gynecologic, head and neck, anorectal, and skin malignancies to establish biomarker predictors for treatment response and identify potential drivers of treatment resistance, which may be undetectable from analyses of only the initial pretreatment biopsy.

\section{Conclusion}

In conclusion, this work provides proof of concept that a noninvasive, swab-based biopsy technique can be utilized to serially sample tumors for in-depth sequencing analysis. This novel methodology can be added to the translational research armamentarium to interrogate tumor genetics and eventually tailor cancer-directed therapies. 


\section{Figures}

\section{Figure 1. Overall Study Design and Quality Characteristics for all Samples by}

Time (A) Patients with cervical cancer underwent five weeks of external beam radiation therapy (EBRT) followed by two brachytherapy treatments (B1 and B2), with swab samples collected at baseline, week 1 , week 3 , and week 5 of radiation therapy. (B) Of the 73 patients accrued on protocol, 70 patients with 218 total samples had DNA of adequate quantity and quality for sequencing. One tumor sample failed sequencing due to low quantity and high degradation and was not included in the analysis. (C) Total DNA concentration recovered did not differ by acquisition timepoint. Total reads (D), mean target coverage $(E)$, and mapping rate $(F)$ did not vary by timepoint.

Figure 2. Computational Pipeline for Whole Exome Sequencing Data. Workflow depicting alignment, variant calling, and data visualization tools and parameters implemented to analyze WES data acquired from tumor DNA collected by cervical swabs.

Figure 3. Top 50 Gene alterations over time for $\mathbf{7 0}$ patients with paired normals. Heat map displaying the top 50 genes ranked by occurrence for 70 patients (217 samples) grouped by timepoint collection during chemoradiation.

Figure 4. Top 50 Gene alterations over time for 33 patients with all four-time points. Heat map displaying the top 50 genes ranked by occurrence for 33 patients (132 samples) grouped by timepoint collection during chemoradiation. 
medRxiv preprint doi: https://doi.org/10.1101/2022.02.23.22271256; this version posted February 24, 2022. The copyright holder for this preprint (which was not certified by peer review) is the author/funder, who has granted medRxiv a license to display the preprint in perpetuity. All rights reserved. No reuse allowed without permission.

\section{Tables}

Table 1. Patient Characteristics

Table 2. Quality Characteristics for Samples by Timepoint

\section{Supplementary Materials}

Table S1. Sample and sequencing quality control metrics and tumor purity estimates for each tumor sample. 
medRxiv preprint doi: https://doi.org/10.1101/2022.02.23.22271256; this version posted February 24, 2022. The copyright holder for this preprint

(which was not certified by peer review) is the author/funder, who has granted medRxiv a license to display the preprint in perpetuity.

All rights reserved. No reuse allowed without permission.

\section{References}

1. Siegel RL, Miller KD, Jemal A. Cancer statistics, 2019. CA: A Cancer Journal for Clinicians. 2019;69(1):7-34. doi:10.3322/caac.21551

2. Arbyn $M$, Weiderpass $E$, Bruni L, et al. Estimates of incidence and mortality of cervical cancer in 2018: a worldwide analysis. Lancet Glob Health. Published online December 4, 2019. doi:10.1016/S2214-109X(19)30482-6

3. zur Hausen H. Papillomaviruses in the causation of human cancers - a brief historical account. Virology. 2009;384(2):260-265. doi:10.1016/j.virol.2008.11.046

4. Monk BJ, Tewari KS, Koh WJ. Multimodality therapy for locally advanced cervical carcinoma: state of the art and future directions. J Clin Oncol. 2007;25(20):29522965. doi:10.1200/JCO.2007.10.8324

5. Schwarz JK, Siegel BA, Dehdashti F, Grigsby PW. Association of posttherapy positron emission tomography with tumor response and survival in cervical carcinoma. JAMA. 2007;298(19):2289-2295. doi:10.1001/jama.298.19.2289

6. Schernberg A, Bockel S, Annede P, et al. Tumor Shrinkage During Chemoradiation in Locally Advanced Cervical Cancer Patients: Prognostic Significance, and Impact for Image-Guided Adaptive Brachytherapy. Int J Radiat Oncol Biol Phys. 2018;102(2):362-372. doi:10.1016/j.jrobp.2018.06.014

7. Ojesina Al, Lichtenstein L, Freeman SS, et al. Landscape of genomic alterations in cervical carcinomas. Nature. 2014;506(7488):371-375. doi:10.1038/nature12881

8. Wu Y, Zhao J, Dong S, et al. Whole-exome and RNA sequencing reveal novel insights into the pathogenesis of HPV associated cervical cancer. Cancer Biomark. 2019;25(4):341-350. doi:10.3233/CBM-190055

9. Cancer Genome Atlas Research Network, Albert Einstein College of Medicine, Analytical Biological Services, et al. Integrated genomic and molecular characterization of cervical cancer. Nature. 2017;543(7645):378-384. doi:10.1038/nature21386

10. Viet $\mathrm{CT}$, Zhang $\mathrm{X}, \mathrm{Xu} \mathrm{K}$, et al. Brush swab as a noninvasive surrogate for tissue biopsies in epigenomic profiling of oral cancer. Biomark Res. 2021;9(1):90. doi:10.1186/s40364-021-00349-x

11. Gissi DB, Morandi L, Colella G, et al. Clinical validation of 13-gene DNA methylation analysis in oral brushing samples for detection of oral carcinoma: Italian multicenter study. Head Neck. 2021;43(5):1563-1573.

doi:10.1002/hed.26624

12. Morandi L, Gissi D, Tarsitano A, et al. DNA methylation analysis by bisulfite nextgeneration sequencing for early detection of oral squamous cell carcinoma and 
medRxiv preprint doi: https://doi.org/10.1101/2022.02.23.22271256; this version posted February 24, 2022. The copyright holder for this preprint

(which was not certified by peer review) is the author/funder, who has granted medRxiv a license to display the preprint in perpetuity.

All rights reserved. No reuse allowed without permission.

high-grade squamous intraepithelial lesion from oral brushing. J Craniomaxillofac Surg. 2015;43(8):1494-1500. doi:10.1016/j.jcms.2015.07.028

13. Srisuttee R, Arayataweegool A, Mahattanasakul P, et al. Evaluation of NID2 promoter methylation for screening of Oral squamous cell carcinoma. BMC Cancer. 2020;20(1):218. doi:10.1186/s12885-020-6692-z

14. Simon $\mathrm{C}$, Chagraoui J, Krosl J, et al. A key role for $\mathrm{EZH} 2$ and associated genes in mouse and human adult T-cell acute leukemia. Genes Dev. 2012;26(7):651-656. doi:10.1101/gad.186411.111

15. Gnirke A, Melnikov A, Maguire J, et al. Solution hybrid selection with ultra-long oligonucleotides for massively parallel targeted sequencing. Nat Biotechnol. 2009;27(2):182-189. doi:10.1038/nbt.1523

16. Li H, Durbin R. Fast and accurate short read alignment with Burrows-Wheeler transform. Bioinformatics. 2009;25(14):1754-1760.

doi:10.1093/bioinformatics/btp324

17. Cibulskis K, Lawrence MS, Carter SL, et al. Sensitive detection of somatic point mutations in impure and heterogeneous cancer samples. Nat Biotechnol.

2013;31(3):213-219. doi:10.1038/nbt.2514

18. Ye K, Schulz MH, Long Q, Apweiler R, Ning Z. Pindel: a pattern growth approach to detect break points of large deletions and medium sized insertions from pairedend short reads. Bioinformatics. 2009;25(21):2865-2871.

doi:10.1093/bioinformatics/btp394

19. Mayakonda A, Lin DC, Assenov Y, Plass C, Koeffler HP. Maftools: efficient and comprehensive analysis of somatic variants in cancer. Genome Res.

2018;28(11):1747-1756. doi:10.1101/gr.239244.118

20. Locallo A, Prandi D, Fedrizzi T, Demichelis F. TPES: tumor purity estimation from SNVs. Bioinformatics. 2019;35(21):4433-4435. doi:10.1093/bioinformatics/btz406

21. Weidhaas JB, Li SX, Winter K, et al. Changes in gene expression predicting local control in cervical cancer: results from Radiation Therapy Oncology Group 0128. Clin Cancer Res. 2009;15(12):4199-4206. doi:10.1158/1078-0432.CCR-08-2257

22. Cosper PF, McNair C, González I, et al. Decreased local immune response and retained HPV gene expression during chemoradiotherapy are associated with treatment resistance and death from cervical cancer. International Journal of Cancer. 2020;146(7):2047-2058. doi:10.1002/ijc.32793

23. Aran D, Sirota M, Butte AJ. Systematic pan-cancer analysis of tumour purity. Nat Commun. 2015;6(1):8971. doi:10.1038/ncomms9971 
medRxiv preprint doi: https://doi.org/10.1101/2022.02.23.22271256; this version posted February 24, 2022. The copyright holder for this preprint

(which was not certified by peer review) is the author/funder, who has granted medRxiv a license to display the preprint in perpetuity.

All rights reserved. No reuse allowed without permission.

24. Yoshihara K, Shahmoradgoli M, Martínez E, et al. Inferring tumour purity and stromal and immune cell admixture from expression data. Nat Commun.

2013;4:2612. doi:10.1038/ncomms3612

25. Carter SL, Cibulskis K, Helman E, et al. Absolute quantification of somatic DNA alterations in human cancer. Nat Biotechnol. 2012;30(5):413-421.

doi:10.1038/nbt.2203

26. Elloumi F, Hu Z, Li Y, et al. Systematic bias in genomic classification due to contaminating non-neoplastic tissue in breast tumor samples. BMC Med Genomics. 2011;4:54. doi:10.1186/1755-8794-4-54

27. Qiao W, Quon G, Csaszar E, Yu M, Morris Q, Zandstra PW. PERT: a method for expression deconvolution of human blood samples from varied microenvironmental and developmental conditions. PLoS Comput Biol. 2012;8(12):e1002838. doi:10.1371/journal.pcbi.1002838

28. Isella C, Terrasi A, Bellomo SE, et al. Stromal contribution to the colorectal cancer transcriptome. Nat Genet. 2015;47(4):312-319. doi:10.1038/ng.3224

29. Favero F, Joshi T, Marquard AM, et al. Sequenza: allele-specific copy number and mutation profiles from tumor sequencing data. Ann Oncol. 2015;26(1):64-70. doi:10.1093/annonc/mdu479 
Figures-Feasibility of a novel non-invasive swab technique for serial whole-exome sequencing of cervical tumors during chemoradiation therapy

Bronk et al. 
A

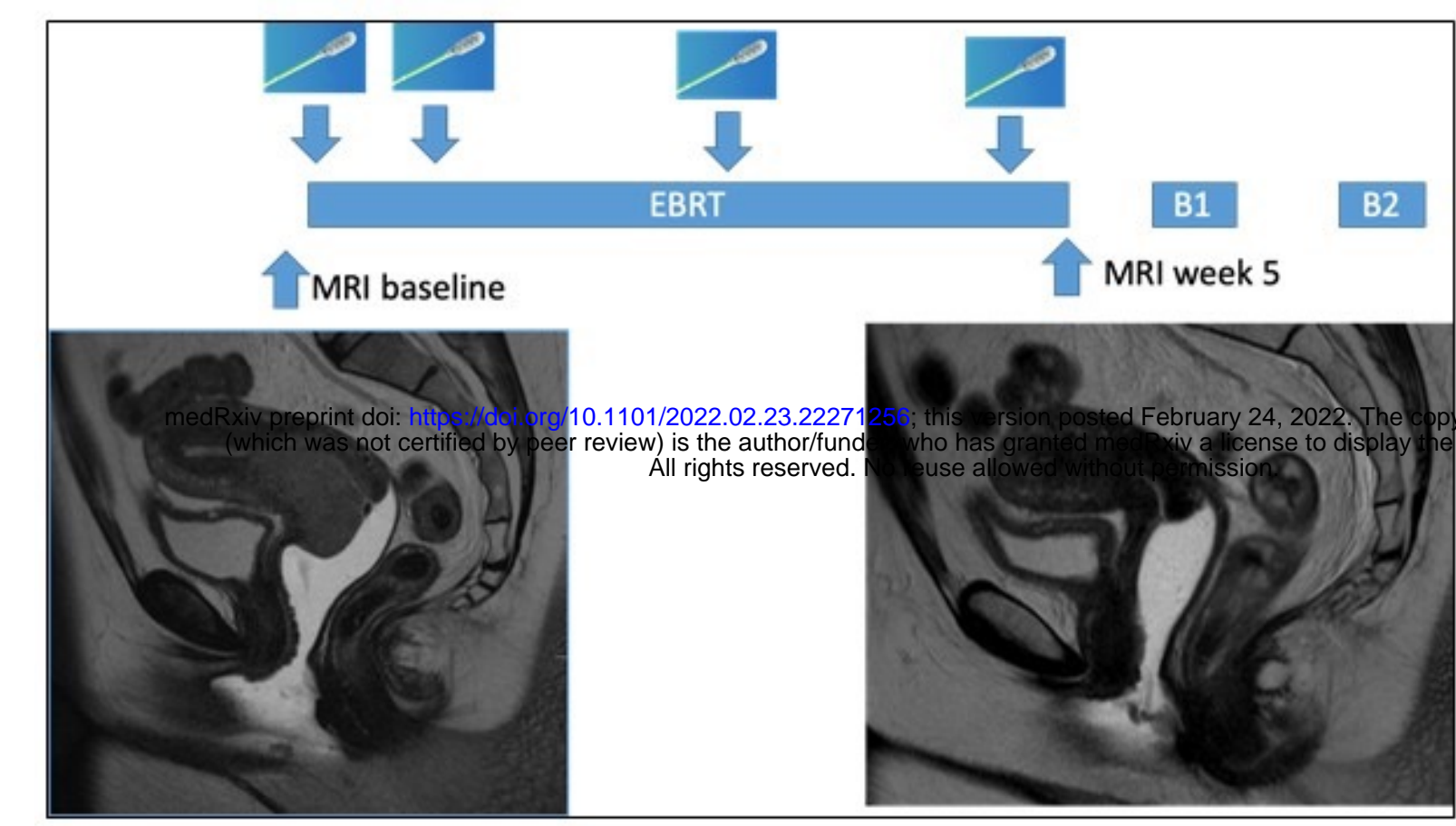

B

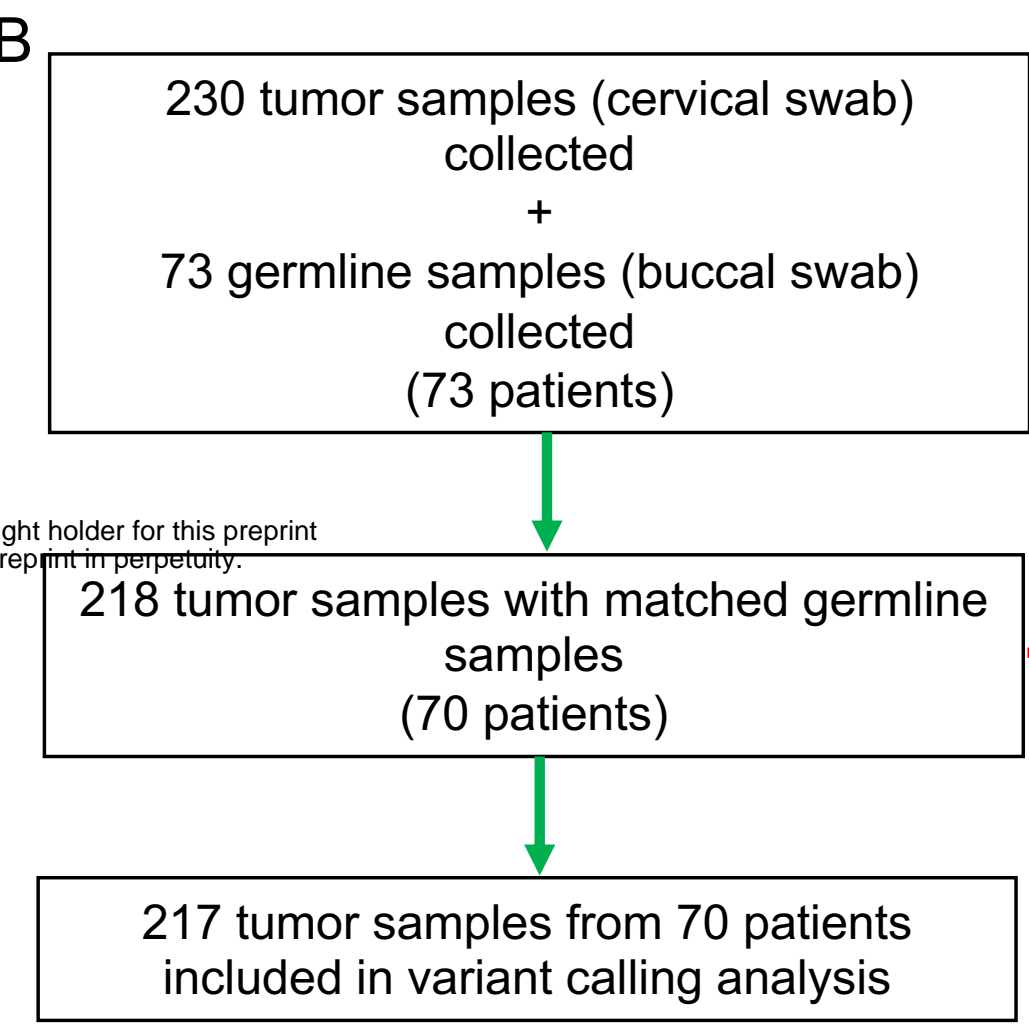

D

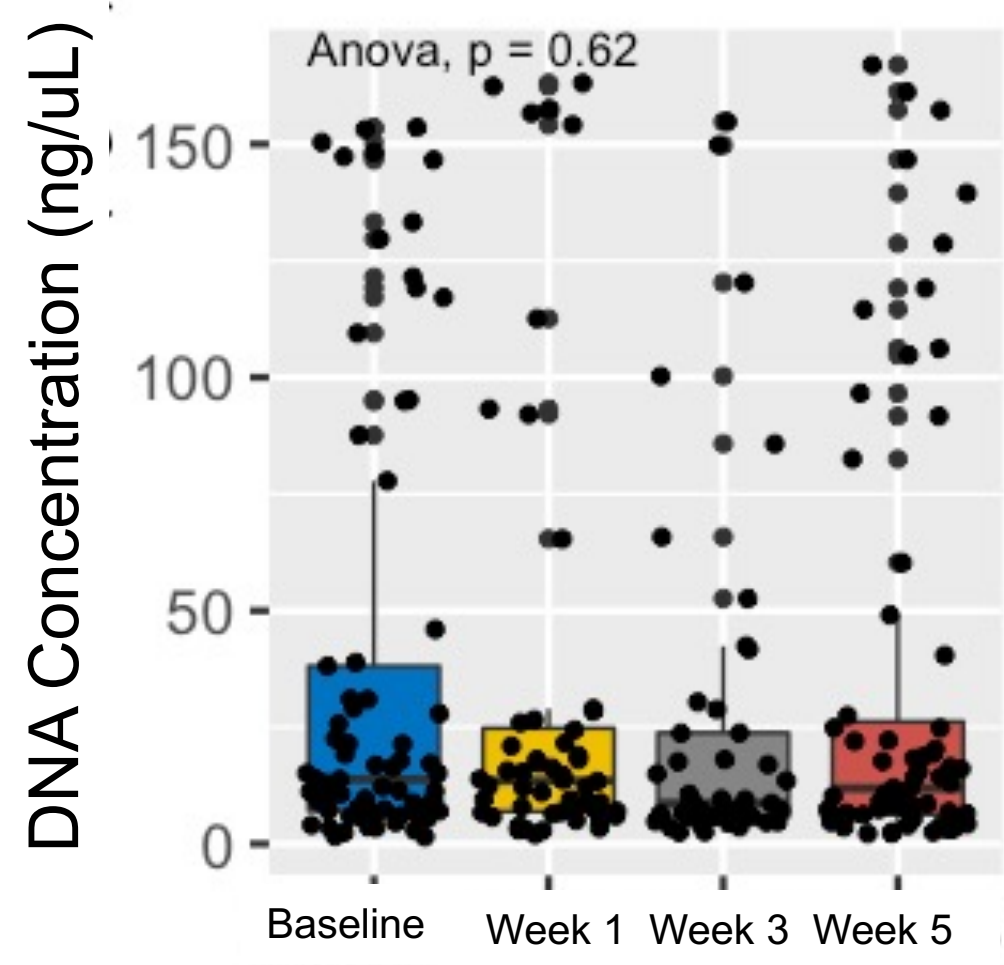

Time

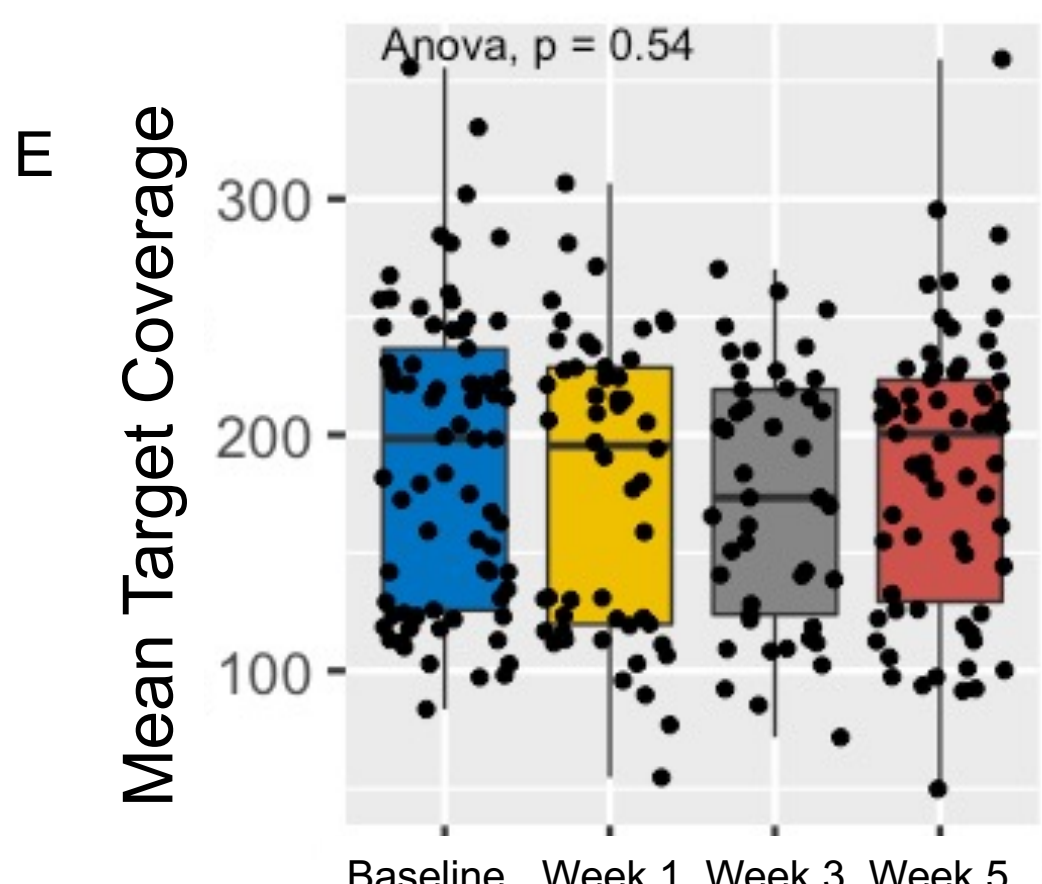

Time

\section{Time \\ † Baseline \\ 由 Week 1 \\ 하 Week 3 \\ 审 Week 5}

Time

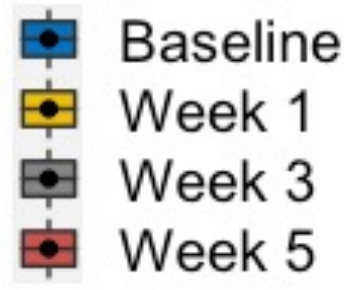

F

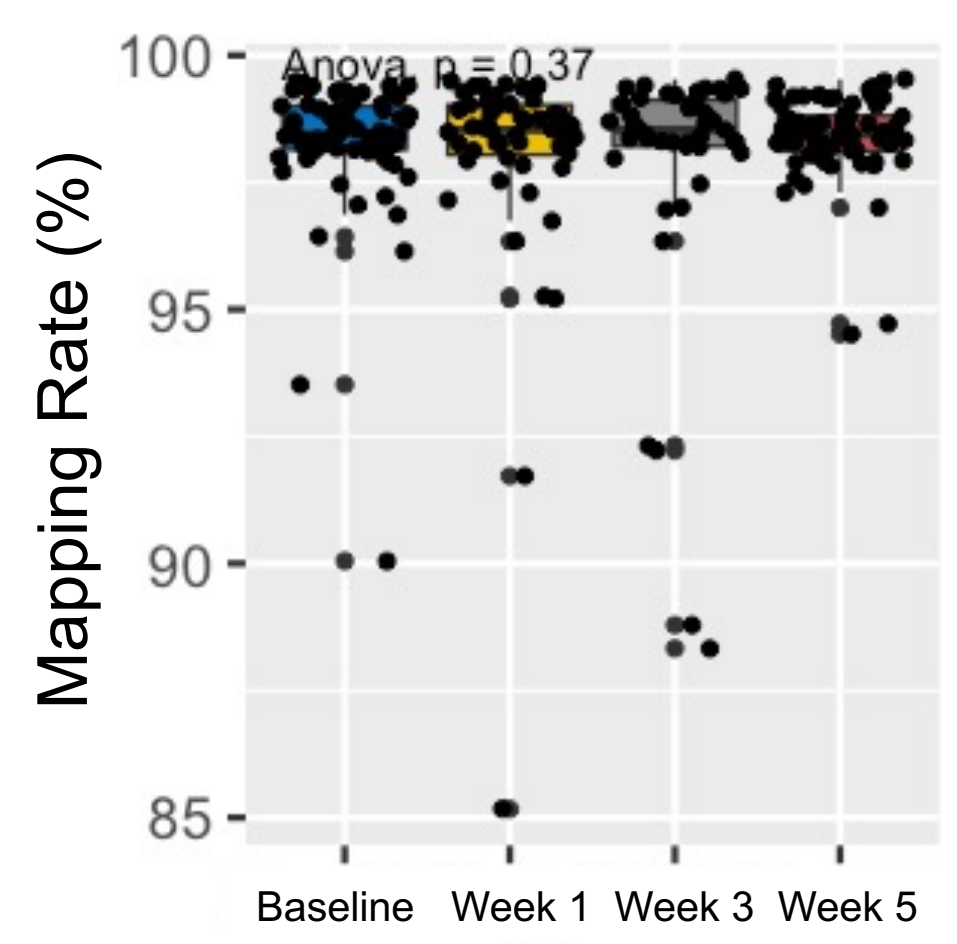
Time

Baseline Week 1 Week 3 Week 5
3 germline samples failed DNA Quality QC; thus 12 matching tumor samples not included (3 patients)

1 tumor sample failed sequencing due to low DNA quantity and high degradation
Time

후 Baseline

क Week 1

由 Week 3

由 Week 5
Time

하 Baseline

由 Week 1

由 Week 3

审 Week 5 


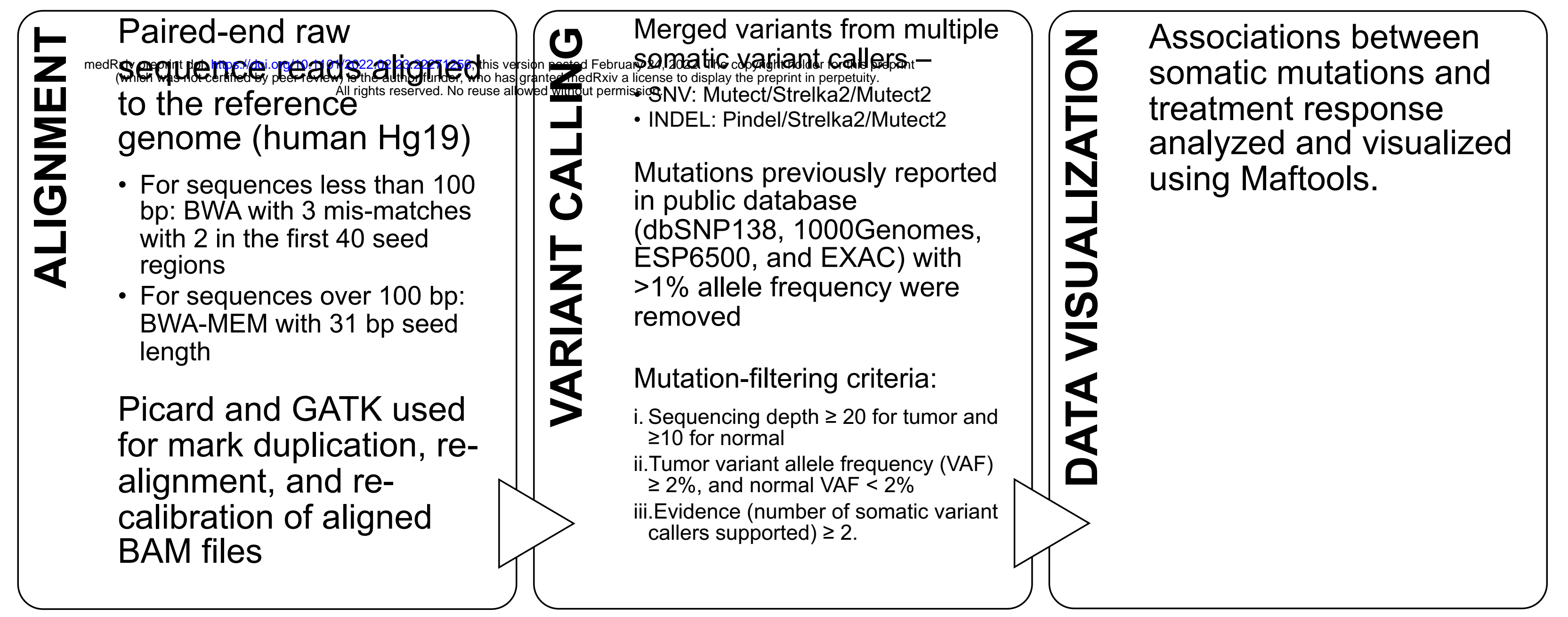


Figure 3

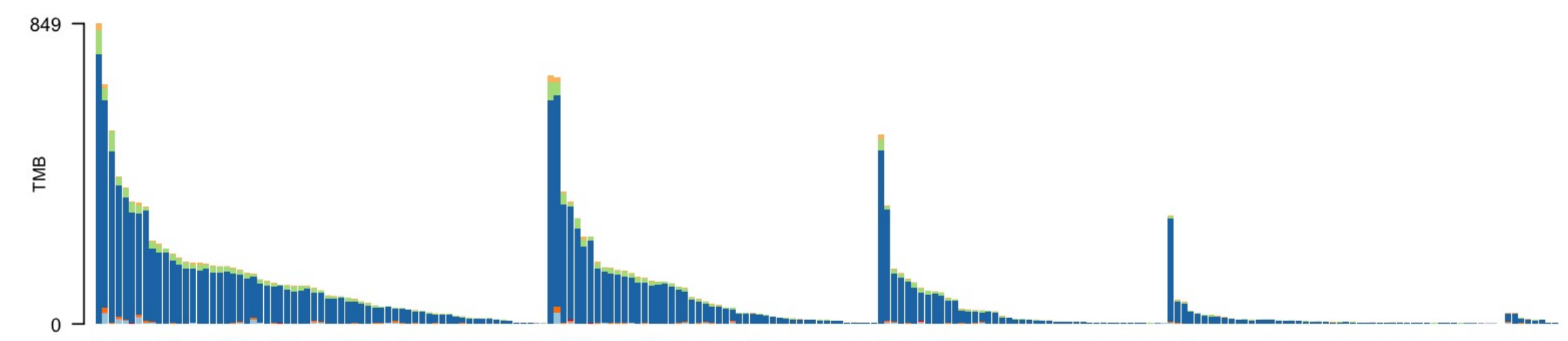

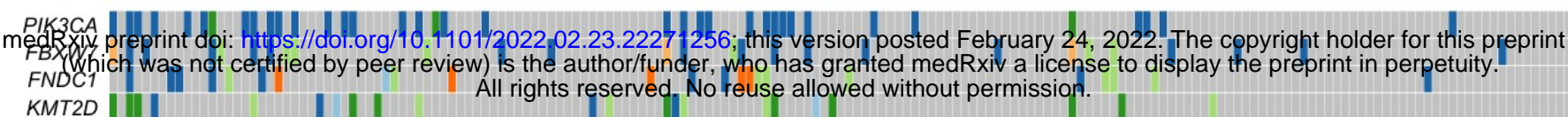

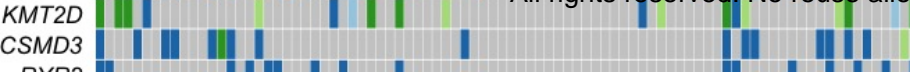
RYR2 MRP1B CGREF1 I OBSCN KMT2C | | | | | | | | | | |

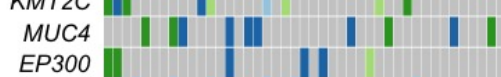
\begin{tabular}{r|}
$E P 300$ \\
$P L E C$ \\
$D N M T 3 A$
\end{tabular}$|\mathbf{|}| \mathbf{|}$

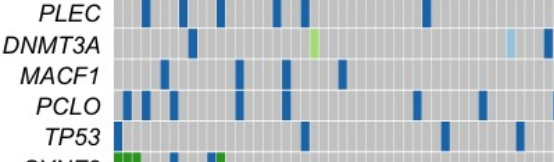
SYNE2 PTEN $S C R I B$
$S M A R C A 4$

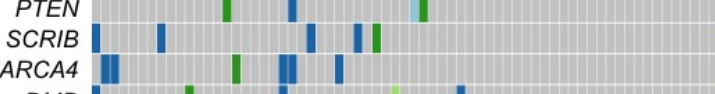

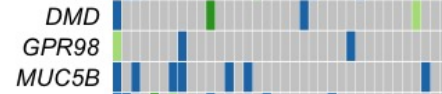
TENM1 | | | | | | | ALMS1 |

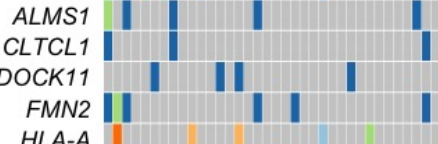
LAMA5 CAMA5 PKHD1L1 | II | NBEA | || | | | | | | | PLCE1

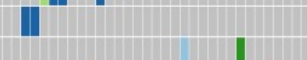

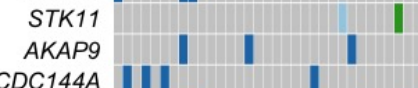

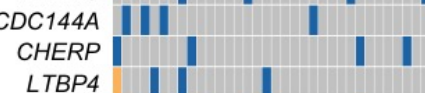

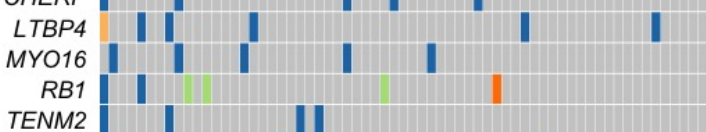

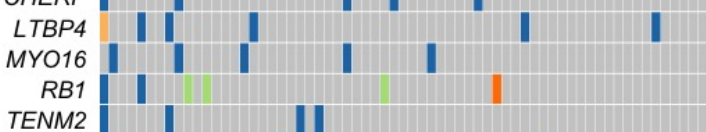

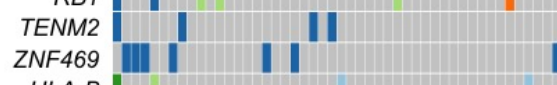
HLA-B HMCN1
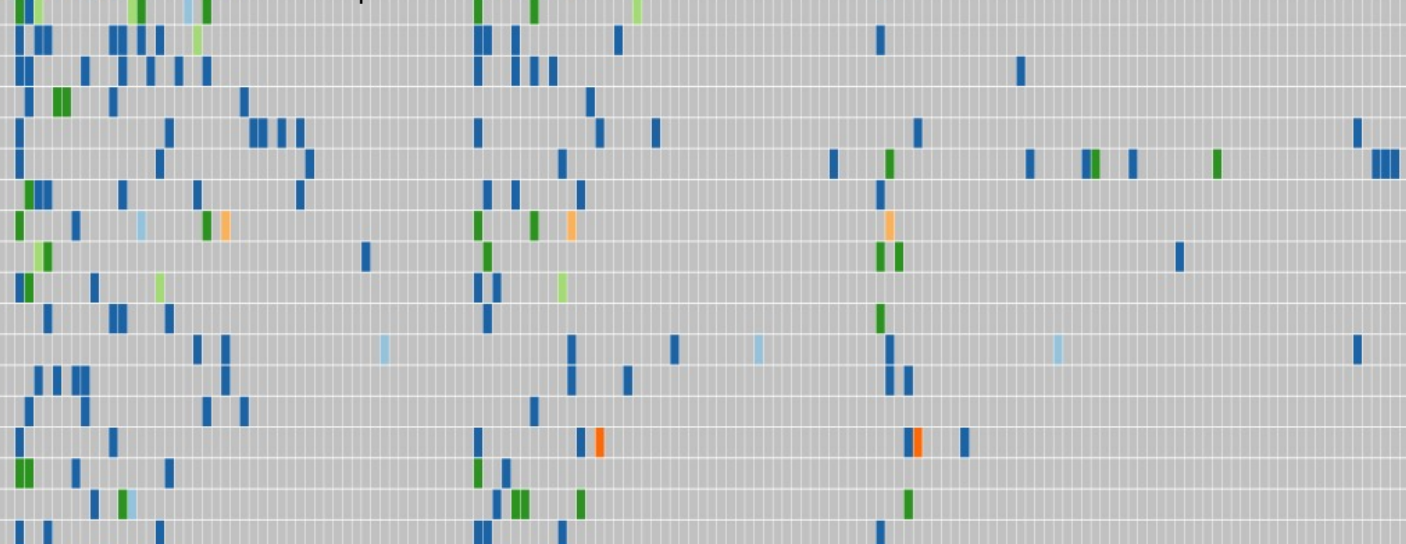

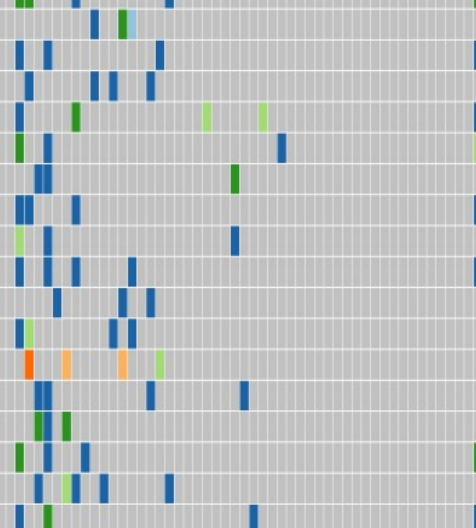
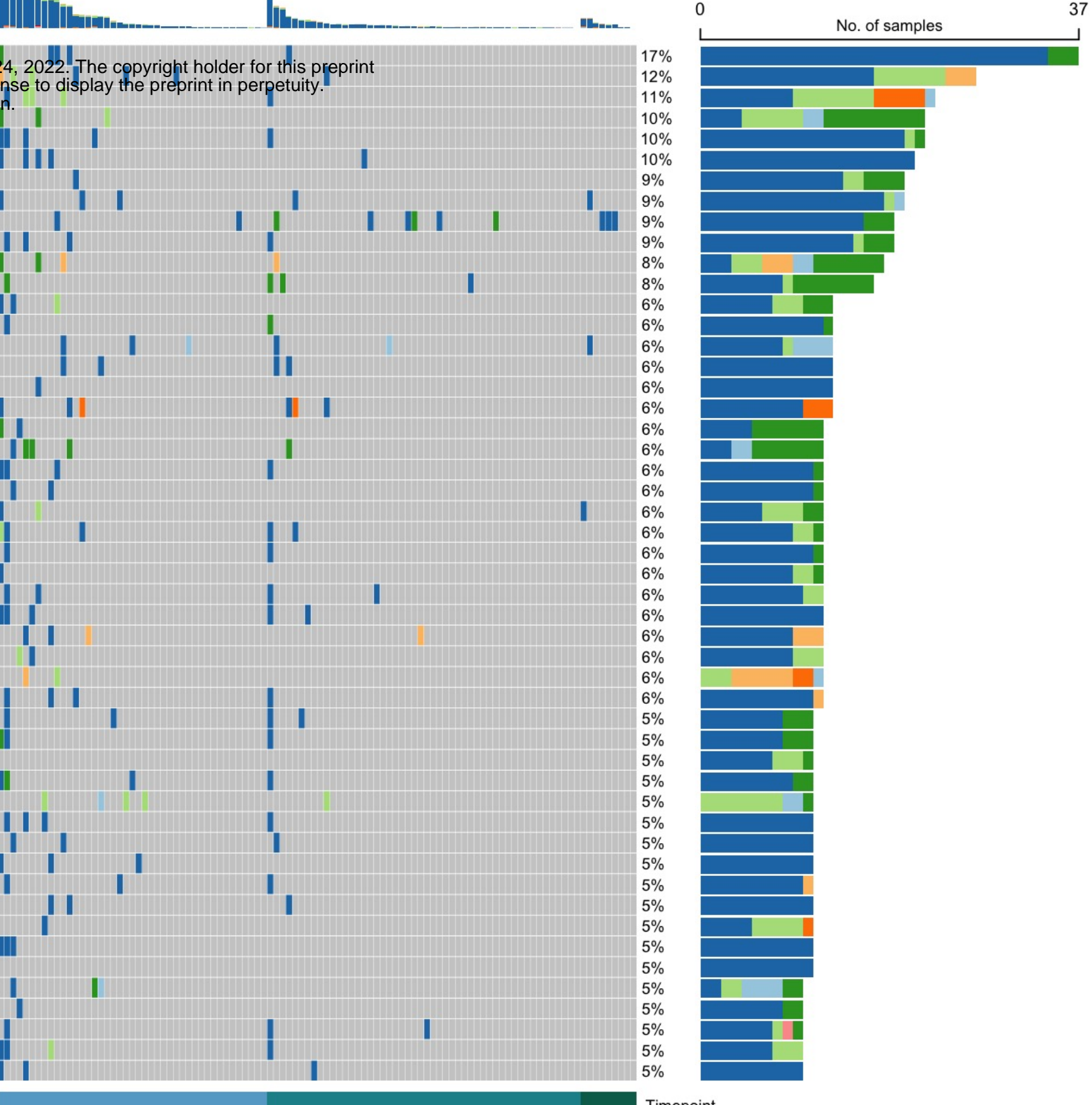

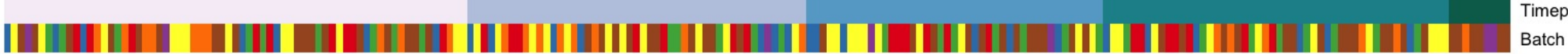

\footnotetext{
- Missense_Mutation

- Frame_Shift_Del

- Nonsense_Mutation

- Frame_Shift_Ins

- Splice_Site

- Multi_Hit

Timepoint

Batch

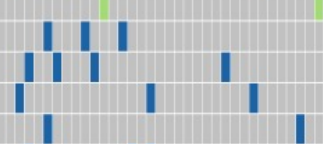

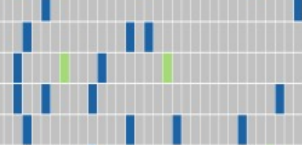
| I I 
Figure 4
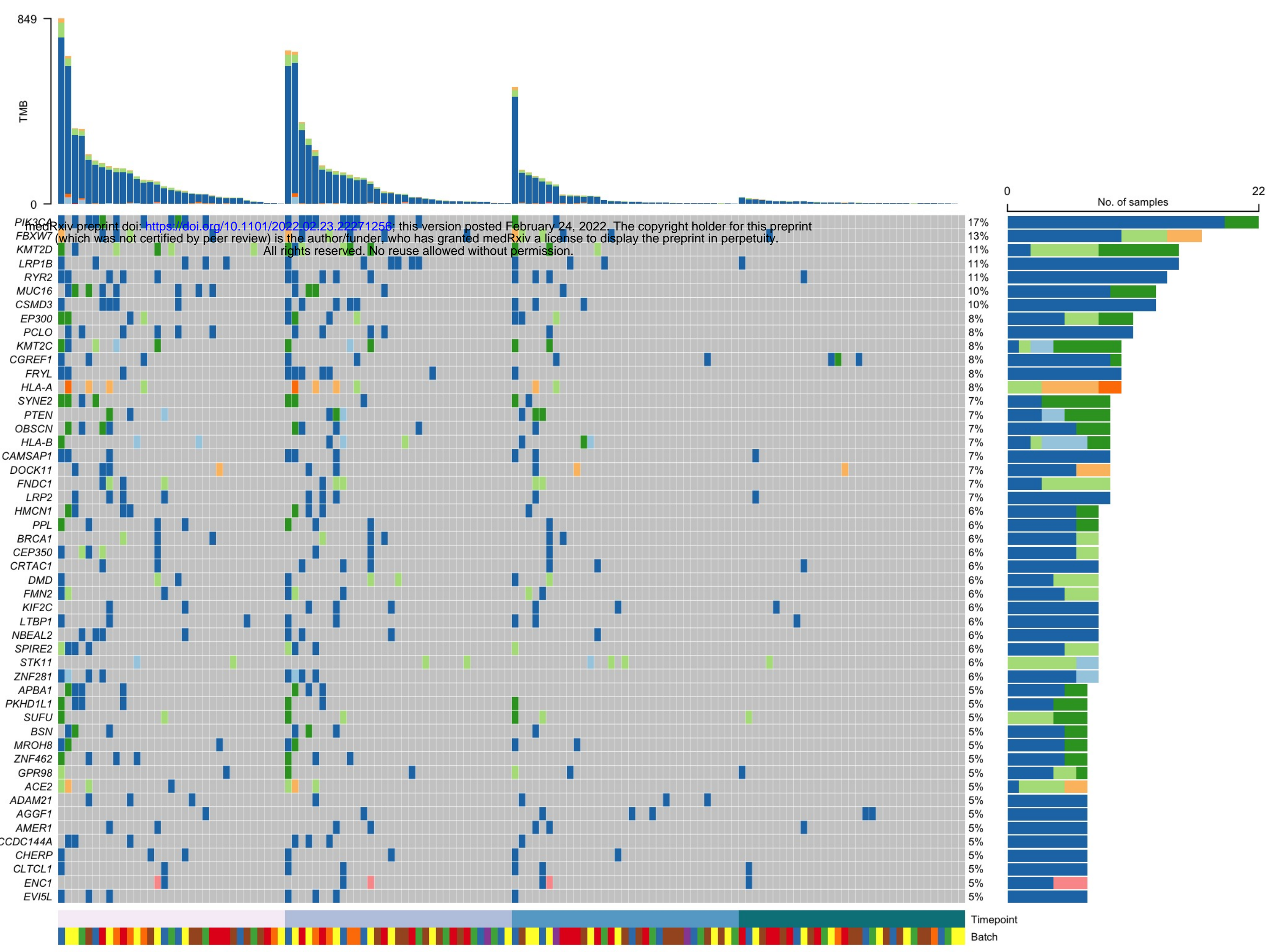

$\begin{array}{ll}\text { - Missense_Mutation } & \text { - } \\ \text { - } \text { Frame_Shift_Ins } \\ \text { - Splice_Site } & \text { - In_Frame_Del } \\ \text { - Frame_Shift_Del } & \text { - Multi_Hit }\end{array}$

Timepoint Batch

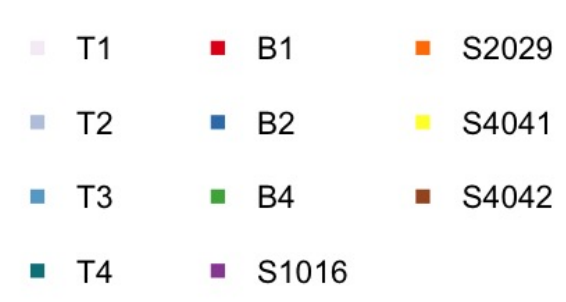


Table 1. Patient Characteristics

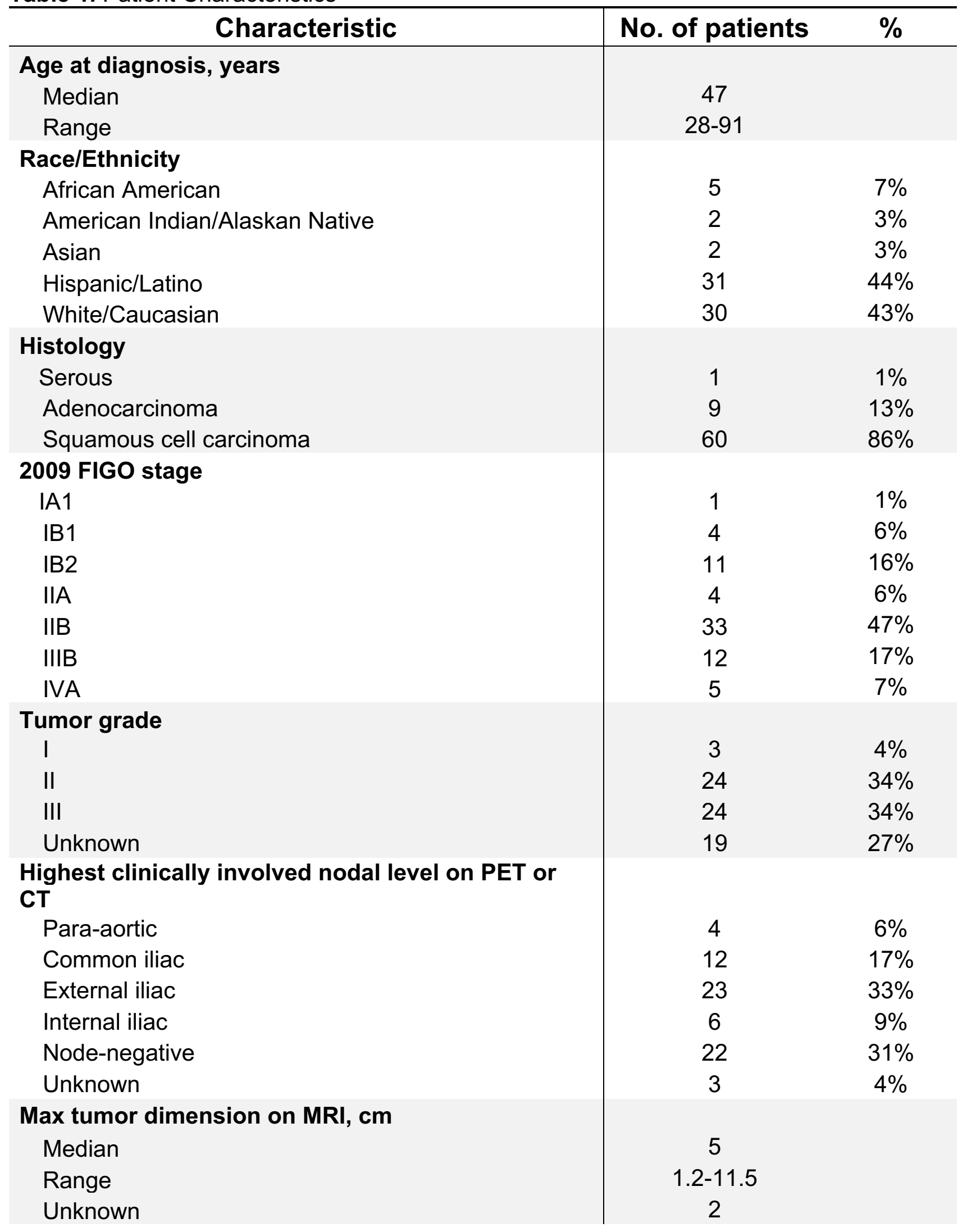


Table 2. Quality Characteristics for Samples by Timepoint

\begin{tabular}{|c|c|c|c|c|}
\hline & Baseline $(\mathrm{N}=70)$ & Week $1(N=49)$ & Week $3(N=42)$ & Week $5(N=61)$ \\
\hline \multicolumn{5}{|c|}{ DNA concentration (ng/uL) } \\
\hline Mean/SD & $37.55 \pm 48.40$ & $32.37 \pm 47.38$ & $25.99 \pm 38.11$ & $34.22 \pm 47.24$ \\
\hline Median & 13.80 & 13.55 & 8.70 & 11.90 \\
\hline \multicolumn{5}{|c|}{ Total Reads (million) } \\
\hline Mean/SD & $224.46 \pm 53.86$ & $219.62 \pm 51.31$ & $212.29 \pm 42.84$ & $220.58 \pm 49.42$ \\
\hline Median & 220.75 & 220.64 & 214.74 & 217.78 \\
\hline \multicolumn{5}{|c|}{ Mapping Rate (\%) } \\
\hline Mean/SD & $98.29 \pm 1.33$ & $98.02 \pm 2.24$ & $97.86 \pm 2.51$ & $98.38 \pm 0.86$ \\
\hline Median & 98.45 & 98.53 & 98.53 & 98.44 \\
\hline \multicolumn{5}{|c|}{ Mean Target Coverage (per sample) } \\
\hline Mean/SD & $189.07 \pm 63.46$ & $179.29 \pm 62.41$ & $173.22 \pm 53.73$ & $184.87 \pm 59.70$ \\
\hline Median & 198.360 & 195.385 & 173.330 & 200.510 \\
\hline \multicolumn{5}{|c|}{ Insertions/ Deletions (Count) } \\
\hline Mean/SD & $42 \pm 20$ & $42 \pm 30.5$ & $39 \pm 29$ & $36 \pm 21$ \\
\hline Median & 38 & 36 & 35 & 32 \\
\hline \multicolumn{5}{|c|}{ Substitutions (Count) } \\
\hline Mean/SD & $296 \pm 402$ & $353 \pm 529$ & $281 \pm 510$ & $124 \pm 241$ \\
\hline Median & 168 & 164 & 137 & 55 \\
\hline \multicolumn{5}{|c|}{ Sequenza Tumor Purity } \\
\hline Mean/SD & $0.28 \pm 0.20$ & $0.27 \pm 0.20$ & $0.23 \pm 0.17$ & $0.22 \pm 0.15$ \\
\hline Median & 0.195 & 0.180 & 0.150 & 0.185 \\
\hline \multicolumn{5}{|c|}{ TPES Tumor Purity } \\
\hline Mean/SD & $0.61 \pm 0.22$ & $0.60 \pm 0.19$ & $0.61 \pm 0.25$ & $0.56 \pm 0.23$ \\
\hline Median & 0.630 & 0.590 & 0.615 & 0.580 \\
\hline
\end{tabular}

